\title{
Evidence of Neuroinflammation and Immunotherapy Responsiveness in Individuals with Down Syndrome Regression Disorder
}

Jonathan Douglas Santoro ( $\nabla$ jdsantoro@chla.usc.edu )

Children's Hospital Los Angeles Department of Pediatrics https://orcid.org/0000-0002-8350-8234

Rebecca Partridge

Virginia Mason Medical Center

Runi Tanna

USC Keck School of Medicine: University of Southern California Keck School of Medicine

Dania Pagarkar

USC Keck School of Medicine: University of Southern California Keck School of Medicine

Mellad Khoshnood

Children's Hospital of Los Angeles

Mustafa Rehmani

Los Angeles County Department of Mental Health

Ryan M. Kammeyer

Children's Hospital Colorado

Grace Y. Gombolay

Emory University School of Medicine

Kristen Fisher

Cincinnati Children's Hospital Medical Center

Allison Conravey

Ochsner Medical Center - New Orleans

Jane El-Dahr

Tulane University School of Medicine

Alison L. Christy

Providence Health

Lina Patel

Children's Hospital Colorado

Melanie A Manning

Stanford University School of Medicine

Heather Van Mater

Duke University School of Medicine

Michael S. Rafii

USC Keck School of Medicine: University of Southern California Keck School of Medicine

Eileen A. Quinn

University of Toledo - Health Science Campus 
Research Article

Keywords: down syndrome, encephalopathy, immunotherapy, cerebrospinal fluid, regression

Posted Date: December 9th, 2021

DOI: https://doi.org/10.21203/rs.3.rs-1135449/v1

License: (c) (i) This work is licensed under a Creative Commons Attribution 4.0 International License. Read Full License 


\section{Abstract \\ Background}

Down syndrome regression disorder is a symptom cluster consisting of neuropsychiatric regression without cause. This study evaluated the incidence of neurodiagnostic abnormalities in individuals with Down syndrome regression disorder and determine if abnormalities are indicative of responses to therapeutic intervention.

\section{Methods}

A retrospective, multi-center, case-control, study was performed. Patients were required to have subacute onset and the presence of four of five symptom groups present (cognitive decline, expressive language, sleep derangement, loss of ability to perform activities of daily living and/or a new movement disorder) and no other explanation for symptoms.

\section{Results}

Individuals with Down syndrome regression disorder were comparable to a cohort of individuals with only Down syndrome although had higher rates of autoimmune disease ( $p=0.02,95 \% \mathrm{Cl}$ : 1.04-1.75). Neurodiagnostic abnormalities were found on EEG $(n=19,26 \%)$, neuroimaging $(n=16,22 \%)$ and CSF $(n=9,17 \%)$. Pleocytosis was appreciated in five cases, elevated total protein in nine, elevated IgG index in seven, and oligoclonal bands in two. Testing within two years of symptom onset were more likely to have neurodiagnostic abnormalities $(p=0.01$, 95\%Cl: 1.64-37.06). In individuals with neurodiagnostic abnormalities immunotherapy was nearly four times more likely to have a therapeutic effect than in those without neurodiagnostic abnormalities (OR: 4.11, 95\% Cl: 1.88-9.02). In those with normal neurodiagnostic studies ( $n=43)$, IVIg was effective in 14 of $17(82 \%)$ patients as well although other immunotherapies were uniformly ineffective.

\section{Conclusions}

This study reports the novel presence of neurodiagnostic testing abnormalities in individuals with Down syndrome regression disorder, providing credence to this symptom cluster potentially being of neurologic and/or neuroimmunologic etiology.

\section{Background}

Down syndrome (DS) is the most common cause of intellectual disability worldwide and occurs in 1 in 800 live births $^{1}$. Neurologic and psychiatric disease in this population is well established; although, over the last decade an increasing frequency of reports of the onset of subacute developmental regression of unclear etiology has been reported $^{2} 3$. This condition has been referred to as Down syndrome disintegrative disorder (DSDD) or Down syndrome regression disorder (DSRD) and "unexplained regression in Down syndrome" (URDS) ${ }^{4}$. Clinical phenomenology consists of a subacute loss of previously acquired developmental skills in the areas of language, communication, cognition, executive function, behavioral, and adaptive skills ${ }^{2-5}$. Other symptoms can include psychiatric manifestations, motor symptoms (stereotypies, extrapyramidal), catatonia, and rapid onset insomnia ${ }^{2}$ 
4-7. DSRD can be severe and significantly impact both the quality of life and autonomy of persons with DS and their families.

Neither epidemiologic studies nor concentrated searches for disease biomarkers have been performed in persons with DSRD, making the etiology of this symptom cluster difficult to ascertain. Neurologic, psychiatric, genetic and neuroimmunologic etiologies have been proposed ${ }^{8}$. A lack of consistent criteria or diagnostic approach to DSRD exists at this time, yielding heterogeneity of diagnostic and neurodiagnostic investigations which has limited generalizability of published reports.

This study sought to investigate the potential role of neurologic and neuroimmunologic dysfunction in persons with DSRD by determining the prevalence of neurodiagnostic abnormalities in persons with DSRD and whether the presence of these findings dictates response to particular therapeutic interventions.

\section{Methods}

\section{Patient Population}

Patients were retrospectively identified following IRB approval through an institution-based audit of in-person evaluations and telemedicine-based consultations for persons with DS and neurocognitive regression from July 1 , 2019, to October 1, 2021. Remote sites (Appendix 1) contributed cases that were similarly identified through course of practice as there is no unique ICD9 or ICD10 code for DSRD.

Inclusion Criteria: All patients were required to have acute or subacute onset of symptoms (defined as 0-12 weeks), a genetically confirmed diagnosis of DS, and an EEG, MRI and lumbar puncture (LP) performed while symptomatic. In addition. As there are no definitive diagnostic criteria for DSRD, patients required at least four of five total clinical features: 1) cognitive decline (defined as any new deficit in memory, processing speed, attention, or awareness), 2) decreased expressive language or mutism, 3) new onset insomnia or hypersomnia, 4) loss of ability to perform activities of daily living, and/or 5) catatonia or a movement disorder (excluding tics).

\section{Exclusion Criteria}

Patients with a history of any prenatal neurologic insult, prematurity (defined as birth < 36 weeks gestation), known/established structural malformation of the brain, history of leukemia/lymphoma, receipt of chemotherapy and/or ionizing radiation, history of neurologic disorder (e.g., infantile spasms), history of complex congenital heart disease requiring surgical intervention (with the exception of ventricular septal defect, atrial septal defect or patent foramen ovale), or untreated thyroid, cardiac, or obstructive sleep apnea (OSA) were excluded. Finally, cases with insufficient data (see neurodiagnostic work up) were also excluded.

\section{Demographic Comparison}

Individuals with DSRD were compared to individuals with only DS. This information was obtained from a multiinstitutional retrospective database of persons with DS and the same exclusion criteria were applied. Inclusion in the comparator group only required a prior diagnosis of DS with enrollment designed to longitudinally follow health outcomes in individuals with DS.

\section{Clinical Assessments and Data Extraction}


All patients were evaluated by a board-certified pediatrician, geneticist, and/or neurologist. Demographic and clinical data were extracted from these encounters and reviewed manually by the authors (JDS, MR, RT) for extraction of established phenotypes in $\mathrm{DSRD}^{4}$. All data was systematically reported and when not documented in the medical record, a query was made to the evaluating clinician for clarification. Symptoms were clustered into eight groups (Appendix 2). The inclusion criteria used in this study included symptoms from this larger grouping although not all symptoms were used in inclusion criteria as both author group and external expert review (acknowledgments) determined that these symptom clusters were best utilized for descriptive, as opposed to diagnostic, purposes. Presence of a symptom cluster in an individual was defined by having $\geq 50 \%$ of the symptoms within any grouping. Each case was reviewed by two authors with a third utilized only when there was discrepant data extracted from a case.

\section{Neurodiagnostic Work Up}

As this study was retrospective in nature, not all neurodiagnostic work ups were executed at the same time points or in the same order. Definitions of EEG, MRI, and LP abnormalities are listed in Appendix 3.

Therapeutic Responses: Quantification of clinical improvement with specific therapeutic interventions was subjectively assessed from documentation by the research team. Clinical response was assessed as any of the following: 1) subjective report by patient, parent, or guardian, 2) resolution of lab or neurodiagnostic study abnormality (e.g., normalization of EEG), 3) discontinuation of medications when clinical improvement was cited as a cause, or 4) improvements on physical examination or neurologic examination (e.g., expressive language documented in a patient with prior mutism). Therapeutic responses were reviewed by two authors with a third utilized only when there was discrepant agreement on a case. Individuals treated with more than one therapy required at least four weeks on treatment without multiple therapeutic changes to be classified as effective/ineffective.

\section{Statistical Analysis}

Descriptive statistics were produced for demographic and clinical presentations. Cohen's kappa was used to assess inter-rater reliability for inclusion/exclusion of cases and in clinical data extraction. Interquartile ranges were calculated for continuous variables. Chi squared analysis was performed for evaluating differences between subgroups. Odds ratios with corresponding $95 \%$ confidence intervals were used to calculate the likelihood of therapeutic benefit between individuals with neurodiagnostic abnormalities and those without. A $p$ nominal value of $<0.05$ was considered statistically significant for each statistical test.

\section{Results}

In total, 97 cases were identified for potential review of which $72(74 \%)$ met criteria for inclusion. The most common reasons for exclusion were incomplete data $(17,68 \%)$, prior diagnosis of a neurologic disorder $(7,28 \%)$, and prior receipt of chemotherapy $(2,8 \%)$. The median number of physician evaluations prior to the diagnosis of DSRD was four, including primary care (IQR: 3-6). Clinical data extracted by the authors yielded a Cohen's kappa coefficient of 0.73 (91\% agreement).

Demographic data are presented in Table 1. The median age of onset was 14 years (IQR: 12-17) with the median age at diagnosis was 19 years (IQR: 13-27) (Figure 1). The majority of this cohort was Caucasian (55, 76\%) with Hispanic ethnicity $(51,71 \%)$, the latter being at a higher rate than our control population $\left(x^{2}(1, N=1289)=50.5\right.$, 
$p<0.001,95 \% \mathrm{Cl}: 1.96-2.76)$. Individuals with DSRD were more likely to have a history of autoimmune disease ( $p=$ $0.02,95 \% \mathrm{Cl}: 1.04-1.75)$ and thyroid disease $(p<0.001,95 \% \mathrm{Cl}: 1.64-3.27)$ compared to individuals with DS without regression.

Clinical phenotypes are presented in Table 1. A potential preceding trigger was observed in 37 cases (51\%) with recent infection reported in 16 cases (43\%). The median time to peak symptoms was three months although this was heterogeneous with an IQR of 1-6.

Serum and neurodiagnostic data are presented in Table 2 and overlap of neurodiagnostic study abnormalities is presented in Figure 2. Serum analysis revealed thyroid peroxidase (TPO) and thyroglobulin antibodies were present in $25(37 \%)$ and 20 (30\%) individuals, respectively, although only TPO antibodies were significantly greater than controls ( $p=0.02,95 \% \mathrm{Cl}$ : 1.06-2.16). Vitamin D 25-OH levels were also significantly lower in individuals with DSRD cohort compared to individuals with DS only $(n=384)(p<0.001,95 \% \mathrm{Cl}: 10.57-16.9)$. Analysis of cytokine profiling revealed abnormalities in 20 individuals (40\%) tested with elevations in soluble IL-2 receptor (13, $62 \%)$ and IL-10 (5, $24 \%)$ most frequently observed. Comparison to individuals with DS without regression was not available for the majority of lab testing reviewed.

Abnormalities in EEG were found in 19 cases (26\%). The most frequently reported electrographic feature were epileptiform discharges in the frontal or temporal lobes $(n=11,58 \%)$. Neuroimaging was abnormal in 16 cases (22\%) with punctate T2 signal abnormalities ( $n=13,81 \%$, Figure 3$)$ and basal ganglia calcification $(n=2,18 \%$, Figure 4) identified. Two individuals were noted to have incidental findings: an anterior temporal arachnoid cyst and Chiari I malformation.

Cerebrospinal fluid was abnormal in nine cases (17\%). Pleocytosis was appreciated in five cases (all with $>90 \%$ lymphocytosis), elevated total protein in nine cases (13\%), elevated IgG index in seven cases (10\%), and oligoclonal bands in two cases (3\%). The Mayo Clinic autoimmune encephalopathy panel was negative in all tested cases in both the serum and CSF ( $n=59)$. Neopterin was elevated in six cases (8\%) although pleocytosis and/or elevated total protein were noted in all as well. In cases where time between diagnosis and neurodiagnostic testing was greater than three years, capture of neurodiagnostic abnormalities was very low (8\%, 2 of 25 patients) compared to patients receiving assessment prior to two years $(40 \%, 19$ of 47 patients) even though clinical presentations were similar $(p=0.01,95 \% \mathrm{Cl}: 1.64-37.06)$.

Clinical symptoms were predictive of some neurodiagnostic study abnormalities (Appendix 4). Predictors of any neurodiagnostic study abnormality included confusion/disorientation ( $p=0.01,95 \% \mathrm{Cl}$ : 1.51-34.67), memory impairment $(p=0.04,95 \% \mathrm{Cl}: 1.07-25.46)$, catatonia $(p=0.04,95 \% \mathrm{Cl}: 1.08-16.21)$, freezing/bradykinesia $(p=0.03$, $95 \% \mathrm{Cl}: 1.04-15.63)$, urinary retention $(p=0.02,95 \% \mathrm{Cl}: 0.02,1.20-9.13)$ as well as the cognitive/executive ( $p=0.01$, $95 \% \mathrm{Cl}: 1.28-5.72)$ and motor symptom clusters ( $p=0.02,95 \% \mathrm{Cl}: 1.09-2.61)$. Low vitamin $\mathrm{D}$ levels were predictive of having any neurodiagnostic abnormalities $\left(r^{2}=0.20, p<0.001,95 \% \mathrm{Cl}: 0.01-0.03\right)$.

Therapeutic interventions and responses are reported in Table 3. Amongst all patients, the most effective therapies were IVIg (38/43, 88\%), benzodiazepines (46/63, 77\%), and electroconvulsive therapy (ECT) (36/49, 74\%). Initial immunotherapeutic interventions were either steroids $(30 \mathrm{mg} / \mathrm{kg} / \mathrm{d}$ for $3-5$ days, max $1000 \mathrm{mg})$ or IVlg (2 g/kg divided over 2-3 days followed by monthly infusions of $1 \mathrm{~g} / \mathrm{kg}$ ) in all cases. IVlg was markedly effective at improving symptoms with 38 (88\%) patients reporting a clinical response as opposed to only 14 (36\%) with steroids. Time to therapeutic effect of steroids or IVIg was rapid at a median of 2.5 weeks (IQR: 1-3) in patients who responded. Although less frequently administered, anti-CD20 therapy (750 mg/m2, max 1000 mg), mycophenolate 
(600 mg/m2, max $2000 \mathrm{mg} / \mathrm{d}$ ) and azathioprine (2 mg/kg/d, max $200 \mathrm{mg} / \mathrm{d}$ ) were also effective in individuals who had both neurodiagnostic abnormalities and a prior response to either steroids or IVIg (Table 3 ).

In individuals with neurodiagnostic abnormalities, use of immunotherapy was nearly four times more likely to have a therapeutic effect compared to individuals without neurodiagnostic abnormalities (OR: $4.11, p=0.001,95 \% \mathrm{Cl}$ : 1.88-9.02). In those with normal neurodiagnostic studies, IVIg was effective in 14 of 17 individuals (82\%) who received it empirically although only one other patient had clinical improvement with other forms of non-steroid immunotherapy (1/14,7\%). Effectiveness of antipsychotics ( $p=0.12,95 \% \mathrm{Cl}$ : $0.12-1.26)$, and benzodiazepines $(p=0.42,95 \% \mathrm{Cl}: 0.23-2.59)$ were not significantly different between groups. Antidepressants and ECT were more likely to be effective in individuals without neurodiagnostic abnormalities compared to those with these findings (OR: 0.20, $p=0.02,95 \% \mathrm{Cl}: 0.05-0.79$ and OR: 0.09, $p=0.04,95 \% \mathrm{Cl}$ : 0.02-0.39, respectively).

\section{Discussion}

This study reports the presence of multiple neurodiagnostic study abnormalities in nearly half of individuals with DSRD and preferential response to immunotherapy in individuals with these confirmed abnormalities. These findings support the possibility that, in a minority of individuals with DSRD, neuroimmunologic and neuroinflammatory etiologies can potentially yield the phenotypic symptom cluster described in the literature. Of note, the rate of neurodiagnostic abnormality capture was markedly higher in individuals receiving diagnostic assessment within two years of symptom onset, highlighting the importance of prompt and aggressive neurodiagnostic work up for this symptom cluster.

The significance of neurodiagnostic study abnormalities in persons with DSRD cannot be understated. Limited case reports in individuals with DSRD have identified CSF anomalies although the variability in these cases made interpretation challenging ${ }^{9}$. In our cohort, $17 \%$ of individuals had some form of CSF abnormality. Although there are no existing normative values for individuals with DS, the presence of CSF pleocytosis ${ }^{10}$, elevated CSF protein ${ }^{10} 11$, presence of restricted oligoclonal bands ${ }^{12}{ }^{13}$, elevated IgG index ${ }^{10}{ }^{13}$, and elevated neopterin ${ }^{14-16}$ have all been linked to the presence of neuroinflammatory disorders in children. The presence of these abnormalities, and the overlap between them and other neurodiagnostic studies, provide preliminary evidence for central nervous system dysfunction in individuals with DSRD. Although neurodiagnostic anomalies were observed, no specific pattern emerged as predictive of the disease or response; yielding the need to interpret all studies as biomarkers of cerebral dysfunction as opposed to being diagnostic or confirmatory of DSRD. Although serum data was of limited value due to low $\mathrm{n}$ in this study, it is noteworthy that that elevations in IL-10 were observed as chromosome 21 encodes for the beta subunit of this interleukin and may provide a fast-forward mechanism of disease in DSRD given that levels of this interleukin have previously been identified as no different than neurotypical individuals ${ }^{17}$.

In our cohort, individuals with DSRD and neurodiagnostic study abnormalities experience a nearly fourfold greater likelihood of response to immunotherapy compared to those without. This observation is particularly striking in that the observed therapeutic response validates the clinical significance of the neurodiagnostic abnormalities observed. The therapeutic response reported builds on prior reports of immunotherapy responsive DSRD in individuals with neurodiagnostic study abnormalties ${ }^{9}{ }^{18}$. Although steroids are well established as first line therapy in a variety of neuro-immunologic disorders ${ }^{19-23}$, efficacy was lower with regards to clinical improvement. The reason for this remains unclear although could potentially be related to heterogeneity in administration (oral versus intravenous) and use in a population likely to experience side effects due to medical comorbidities which may 
obscure clinical improvement. Amongst individuals who responded to IVIg, use of other immunotherapeutics was nearly uniformly beneficial in individuals with or without neurodiagnostic study abnormalities. This highlights the possibility that while IVIg may be beneficial for the treatment of DSRD, primary neuroinflammation is not likely present in all cases, requiring a more nuanced assessment of need for second line immunotherapy by physicians.

Individuals without neurodiagnostic abnormalities were twice as likely to respond to antipsychotics, five times more likely to respond to anti-depressants, and ten times more likely to respond to ECT, the latter two being statistically significant differences. DSRD is a symptom cluster, and thus, multiple etiologies for this condition are highly likely. These findings provide preliminary evidence that distinct etiologies of DSRD may respond differently to therapeutic interventions, highlighting the need for thorough clinical assessment and neurodiagnostic study obtainment. Further supporting this concept is the identification that all individuals with DSRD onset $<8$ years were not responsive to immunotherapy.

Assessing CNS inflammation and autoimmunity poses many challenges, including current testing modalities that are insufficient to capture the breadth of immune mediated processes. Ongoing discoveries in the area of autoimmune encephalitis (AE) have highlighted these limitations and revealed that a substantial number of cases may have unremarkable neurodiagnostic studies during their disease course, regardless of disease activity $24-26$. While the overall response to immunotherapy was lower in individuals without neurodiagnostic abnormalities in our cohort, it continues to remain possible that capture of neurodiagnostic abnormalities may be time-dependent, which was appreciated in the lower yield of testing abnormalities in individuals with longer time between symptom onset and diagnosis. Thus, it could be argued that obtainment of neurodiagnostic studies could be more beneficial for guiding second line immunotherapy than for initiation of primary immunotherapeutics such as steroids and IVIg, which carry a low side effect profile and marked efficacy in sub-both groups. However, the data builds upon prior reports ${ }^{918}$ of use of immunotherapy in individuals with DSRD regardless of neurodiagnostic study results.

A question that emerges from this study is whether DSRD is a form of AE given the temporal and symptomatic overlap between the conditions ${ }^{19} 27$. Lack of definitive autoantibody capture and a phenotype unlike any established form of $A E$ that has been identified argue for a distinct entity ${ }^{28}$. In addition, younger patients have a much higher rate of seizure in the setting of autoimmune encephalitis which was not reported in this cohort ${ }^{10} 19$. Finally, when utilizing Graus et al (2016) criteria ${ }^{27}$, only four patients (6\%) met criteria for autoantibody-negative but probable AE. It is the opinion of the authors that in a minority of individuals with DSRD, there may be similar neuroinflammatory mechanisms at play as in AE although ultimately, this process is likely unique.

This study is not without limitations. Firstly, the retrospective nature of this study introduces selection bias and recall bias. While the authors attempted to mitigate this by having very strict inclusion/exclusion criteria, this also reduced the total " $n$ " for this study, which already reports a rare disease entity. Most individuals were evaluated in neuroimmunology clinics, introducing potential for ascertainment and confirmation. This study required substantive subjective review of data for analysis although this was controlled using a two-tiered review system with neutral arbiter. The subjective nature of report and lack of specific grading systems or diagnostic criteria for DSRD did make this challenging. Our study's high Cohen's kappa (0.72) indicated substantial agreement between reviewers, lowering the risk of misinterpretation of data by single reviewers. Further, efficacy was interpreted broadly, and particular therapies may have resulted in isolated symptomatic improvement of symptoms as opposed to the underlying disease process (e.g., benzodiazepines improving catatonia but not DSRD). Another confounder is that the median time from onset to evaluation in this study was two years. This may have caused a 
higher likelihood to detect some neurodiagnostic abnormalities (EEG/MRI) and lower likelihood to detect others (CSF). The restrictive inclusion/exclusion criteria of this study may have also elevated the rate of capture for neurodiagnostic abnormalities by way of excluding patients with a lower likelihood neurologic disease. Finally, work ups were heterogeneous in this cohort which limits the power and generalizability of some of the findings reported, especially in that there are not well-studied reference ranges for individuals with DS without DSRD for many of these labs.

\section{Conclusions}

This study reports the novel presence of electrographic, neuroimaging and CSF testing abnormalities in individuals with DSRD, providing credence to this symptom cluster being neurologic and/or neuroimmunologic in origin in a minority of cases. Given the importance of identifying a potential etiology to this disease and the marked therapeutic effect of immunotherapy in persons with neurodiagnostic abnormalities, further study into the role of neuroinflammation in DSRD is warranted and desperately needed.

\section{Abbreviations}

AE: autoimmune encephalitis

CSF: cerebrospinal fluid

DS: Down syndrome

DSDD: Down syndrome disintegrative disorder

DSRD: Down syndrome regression disorder

ECT: electroconvulsive therapy

EEG: electroencephalogram

LP: lumbar puncture

IVIg: intravenous immunoglobulin

MRI: magnetic resonance imaging

OSA: obstructive sleep apnea

URDS: unexplained regression in Down syndrome

\section{Declarations}

Ethics Approval: This study was IRB approved. Consent and assent were waived as it was retrospective in nature.

Competing Interests: The authors report no financial or non-financial competing interests.

Funding: This study was not funded. 
Data Availability. Anonymized data will be made available to qualified investigators upon request and IRB approval for release.

Acknowledgments: The authors wish to thank the Down syndrome program at Massachusetts General Hospital and specifically Drs. Stephanie Santoro and Brian Skotko for their assistance with review of this manuscript and collaboration of research efforts. The authors would also like to thank the Byrne family for their generous contributions to research in Down syndrome regression disorder.

Author Contributions: Drs. Santoro, Rafii and Quinn conceptualized and designed the study, designed inclusion/exclusion criteria, performed the analysis, drafted the initial manuscript, performed data review, and reviewed and revised the manuscript. Drs. Tanna, Pagarkar, Khoshnood, and Rehmani performed data entry and extraction, served as auditors of therapeutic responses, assisted with data integrity review, and reviewed and revised the manuscript. Drs. Patridge, Kammeyer, Gombolay, Fisher, Conravey, El-Dahr, Christy, Patel, Manning, and Van Matter collaborated on cases, performed data entry, and reviewed, edited, and revised the manuscript. All authors approved the final manuscript as submitted and agree to be accountable for all aspects of the work.

Author Information: not applicable

Consent for Publication: not applicable

\section{References}

1. de Graaf G, Buckley F, Skotko BG. Estimation of the number of people with Down syndrome in Europe. Eur J Hum Genet 2021;29(3):402-10. doi: 10.1038/s41431-020-00748-y [published Online First: 2020/11/02]

2. Worley G, Crissman BG, Cadogan E, et al. Down Syndrome Disintegrative Disorder: New-Onset Autistic Regression, Dementia, and Insomnia in Older Children and Adolescents With Down Syndrome. J Child Neurol 2015;30(9):1147-52. doi: 10.1177/0883073814554654 [published Online First: 2014/11/05]

3. Mircher C, Cieuta-Walti C, Marey I, et al. Acute Regression in Young People with Down Syndrome. Brain Sci 2017;7(6) doi: 10.3390/brainsci7060057 [published Online First: 2017/05/31]

4. Santoro SL, Cannon S, Capone G, et al. Unexplained regression in Down syndrome: 35 cases from an international Down syndrome database. Genet Med 2020;22(4):767-76. doi: 10.1038/s41436-019-0706-8 [published Online First: 2019/11/27]

5. Ghaziuddin N, Nassiri A, Miles JH. Catatonia in Down syndrome; a treatable cause of regression. Neuropsychiatr Dis Treat 2015;11:941-9. doi: 10.2147/ndt.S77307 [published Online First: 2015/04/22]

6. Akahoshi K, Matsuda H, Funahashi M, et al. Acute neuropsychiatric disorders in adolescents and young adults with Down syndrome: Japanese case reports. Neuropsychiatr Dis Treat 2012;8:339-45. doi:

10.2147/ndt.S32767 [published Online First: 2012/08/14]

7. Stein DS, Munir KM, Karweck AJ, et al. Developmental Regression, Depression, and Psychosocial Stress in an Adolescent with Down Syndrome. J Dev Behav Pediatr 2017;38 Suppl 1(Suppl 1):S26-s28. doi: 10.1097/dbp.0000000000000401 [published Online First: 2017/02/01]

8. Rosso M, Fremion E, Santoro SL, et al. Down Syndrome Disintegrative Disorder: A Clinical Regression Syndrome of Increasing Importance. Pediatrics 2020;145(6) doi: 10.1542/peds.2019-2939 [published Online First: 2020/05/31] 
9. Cardinale KM, Bocharnikov A, Hart SJ, et al. Immunotherapy in selected patients with Down syndrome disintegrative disorder. Dev Med Child Neurol 2019;61(7):847-51. doi: 10.1111/dmcn.14127 [published Online First: 2018/12/15]

10. Roliz A, Shah Y, Morse A, et al. Clinical features of paediatric and adult autoimmune encephalitis: A multicenter sample. Eur J Paediatr Neuro/ 2021;30:82-87. doi: 10.1016/j.ejpn.2021.01.001 [published Online First: 2021/01/19]

11. Jarius S, Lechner C, Wendel EM, et al. Cerebrospinal fluid findings in patients with myelin oligodendrocyte glycoprotein (MOG) antibodies. Part 2: Results from 108 lumbar punctures in 80 pediatric patients. $J$ Neuroinflammation 2020;17(1):262. doi: 10.1186/s12974-020-01825-1 [published Online First: 2020/09/05]

12. Sinclair AJ, Wienholt L, Tantsis E, et al. Clinical association of intrathecal and mirrored oligoclonal bands in paediatric neurology. Dev Med Child Neuro/ 2013;55(1):71-5. doi: 10.1111/j.1469-8749.2012.04443.x [published Online First: 2012/10/26]

13. Hacohen $Y$, Singh R, Forsyth V, et al. CSF albumin and immunoglobulin analyses in childhood neurologic disorders. Neurol Neuroimmunol Neuroinflamm 2014;1(1):e10. doi: 10.1212/nxi.0000000000000010 [published Online First: 2014/10/24]

14. Dale RC, Brilot F, Fagan E, et al. Cerebrospinal fluid neopterin in paediatric neurology: a marker of active central nervous system inflammation. Dev Med Child Neurol 2009;51(4):317-23. doi: 10.1111/j.14698749.2008.03225.x [published Online First: 2009/02/05]

15. Molero-Luis M, Casas-Alba D, Orellana G, et al. Cerebrospinal fluid neopterin as a biomarker of neuroinflammatory diseases. Sci Rep 2020;10(1):18291. doi: 10.1038/s41598-020-75500-z [published Online First: 2020/10/28]

16. Molero-Luis M, Fernández-Ureña S, Jordán I, et al. Cerebrospinal fluid neopterin analysis in neuropediatric patients: establishment of a new cut off-value for the identification of inflammatory-immune mediated processes. PLoS One 2013;8(12):e83237. doi: 10.1371/journal.pone.0083237 [published Online First: 2013/12/25]

17. Tarani L, Carito V, Ferraguti G, et al. Neuroinflammatory Markers in the Serum of Prepubertal Children with Down Syndrome. J Immunol Res 2020;2020:6937154. doi: 10.1155/2020/6937154 [published Online First: 2020/04/14]

18. Hart SJ, Worley G, Kishnani PS, et al. Case Report: Improvement Following Immunotherapy in an Individual With Seronegative Down Syndrome Disintegrative Disorder. Front Neurol 2021;12:621637. doi: 10.3389/fneur.2021.621637 [published Online First: 2021/04/13]

19. Lee S, Kim HD, Lee JS, et al. Clinical Features and Treatment Outcomes of Seronegative Pediatric Autoimmune Encephalitis. J Clin Neurol 2021;17(2):300-06. doi: 10.3988/jcn.2021.17.2.300 [published Online First: 2021/04/10]

20. McGetrick ME, Varughese NA, Miles DK, et al. Clinical Features, Treatment Strategies, and Outcomes in Hospitalized Children With Immune-Mediated Encephalopathies. Pediatr Neurol 2021;116:20-26. doi: 10.1016/j.pediatrneurol.2020.11.014 [published Online First: 2021/01/04]

21. Shim Y, Kim SY, Kim H, et al. Clinical outcomes of pediatric Anti-NMDA receptor encephalitis. Eur J Paediatr Neurol 2020;29:87-91. doi: 10.1016/j.ejpn.2020.10.001 [published Online First: 2020/10/14]

22. Bruijstens AL, Wendel EM, Lechner C, et al. E.U. paediatric MOG consortium consensus: Part 5 - Treatment of paediatric myelin oligodendrocyte glycoprotein antibody-associated disorders. Eur J Paediatr Neurol 
2020;29:41-53. doi: 10.1016/j.ejpn.2020.10.005 [published Online First: 2020/11/13]

23. Mizia-Malarz A, Stolpa W, Sobol-Milejska G. The Treatment of Opsoclonus-Myoclonus Syndrome Secondary to Neuroblastic Tumours-Single-Centre Experience and Literature Review. Medicina (Kaunas) 2020;56(8) doi: 10.3390/medicina56080412 [published Online First: 2020/08/23]

24. Hébert J, Gros P, Lapointe S, et al. Searching for autoimmune encephalitis: Beware of normal CSF. J Neuroimmuno/ 2020;345:577285. doi: 10.1016/j.jneuroim.2020.577285 [published Online First: 2020/06/21]

25. Zrzavy T, Höftberger R, Wimmer I, et al. Longitudinal CSF Findings in Autoimmune Encephalitis-A Monocentric Cohort Study. Front Immunol 2021;12:646940. doi: 10.3389/fimmu.2021.646940 [published Online First: 2021/04/09]

26. Albert DV, Pluto CP, Weber A, et al. Utility of Neurodiagnostic Studies in the Diagnosis of Autoimmune Encephalitis in Children. Pediatr Neurol 2016;55:37-45. doi: 10.1016/j.pediatrneurol.2015.10.016 [published Online First: 2016/01/05]

27. Graus F, Titulaer MJ, Balu R, et al. A clinical approach to diagnosis of autoimmune encephalitis. Lancet Neurol 2016;15(4):391-404. doi: 10.1016/s1474-4422(15)00401-9 [published Online First: 2016/02/26]

28. Cellucci T, Van Mater H, Graus F, et al. Clinical approach to the diagnosis of autoimmune encephalitis in the pediatric patient. Neurol Neuroimmunol Neuroinflamm 2020;7(2) doi: 10.1212/nxi.0000000000000663 [published Online First: 2020/01/19]

\section{Tables}

Table 1: Demographics and clinical features 


\begin{tabular}{|c|c|c|c|c|}
\hline & $\begin{array}{l}\text { DSRD } \\
(n=72)\end{array}$ & $\begin{array}{l}\text { DS } \\
(n=1217)\end{array}$ & $p$ value & $95 \% \mathrm{Cl}$ \\
\hline \multicolumn{5}{|l|}{ Sex } \\
\hline Male & $35(49 \%)$ & $578(47.5 \%)$ & 0.85 & $0.80-1.31$ \\
\hline Female & $37(51 \%)$ & $638(52.5 \%)$ & & \\
\hline \multicolumn{5}{|l|}{ Race } \\
\hline Caucasian & $55(76 \%)$ & $1058(87 \%)$ & 0.06 & $0.77-1.01$ \\
\hline Asian & $10(14 \%)$ & $109(9 \%)$ & 0.15 & $0.85-2.83$ \\
\hline Black & $7(10 \%)$ & $49(4 \%)$ & 0.22 & $0.13-5.14$ \\
\hline Hispanic Ethnicity & $51(71 \%)$ & $370(35 \%)$ & $<0.001$ & $1.96-2.76$ \\
\hline \multicolumn{5}{|l|}{ Medical History } \\
\hline Obstructive sleep apnea $(n=70)$ & $35(50 \%)$ & $523(43 \%)$ & 0.22 & $0.91-1.48$ \\
\hline Congenital heart disease & $38(53 \%)$ & $548(45 \%)$ & 0.12 & $0.96-1.51$ \\
\hline Autoimmune disease (any) & $33(46 \%)$ & $426(35 \%)$ & 0.02 & $1.04-1.75$ \\
\hline Thyroid disease & $25(34 \%)$ & $182(15 \%)$ & $<0.001$ & $1.64-3.27$ \\
\hline Age at Onset (median, IQR) & $14(12-17)$ & $11(7-15)^{a}$ & $<0.001$ & $1.05-3.55$ \\
\hline Preceding Trigger & $37(51 \%)$ & & & \\
\hline Infection $^{b}$ & $16(43 \%)$ & & & \\
\hline Change in school/work/home environment & $10(27 \%)$ & & & \\
\hline Loss of family/caregiver/friend & $4(10 \%)$ & & & \\
\hline Change in residence & $2(5 \%)$ & & & \\
\hline Abuse & $2(5 \%)$ & & & \\
\hline Death in immediate social network & $2(5 \%)$ & & & \\
\hline Medical change & $1(3 \%)$ & & & \\
\hline Months to Symptom Peak (median, IQR) & $3(1-6)$ & & & \\
\hline \multicolumn{5}{|l|}{ Clinical Symptoms } \\
\hline Social withdrawal & $72(100 \%)$ & & & \\
\hline Loss of acquired skills & $72(100 \%)$ & & & \\
\hline New autistic features & $72(100 \%)$ & & & \\
\hline Diminished eye contact & $69(96 \%)$ & & & \\
\hline Apathy $(n=70)$ & $66(94 \%)$ & & & \\
\hline Impaired attention & $64(89 \%)$ & & & \\
\hline
\end{tabular}




\begin{tabular}{|c|c|}
\hline Anxiety & $62(86 \%)$ \\
\hline Mutism/Expressive language regression & $59(82 \%)$ \\
\hline Insomnia & $58(81 \%)$ \\
\hline Anorexia & $56(78 \%)$ \\
\hline Whispered speech & $56(78 \%)$ \\
\hline Confusion/disorganization & $55(76 \%)$ \\
\hline Memory impairment & $55(76 \%)$ \\
\hline Catatonia & $55(76 \%)$ \\
\hline Freezing/bradykinesia & $52(72 \%)$ \\
\hline Abulia/avolition $(n=71)$ & $46(65 \%)$ \\
\hline Emotional lability $(n=61)$ & $37(61 \%)$ \\
\hline Stereotypy & $38(53 \%)$ \\
\hline Urinary retention & $38(53 \%)$ \\
\hline Incontinence (urine/feces) & $36(50 \%)$ \\
\hline Inappropriate/mirthless laughter $(n=65)$ & $34(52 \%)$ \\
\hline Circadian rhythm alteration & $26(36 \%)$ \\
\hline New obsessive-compulsive tendencies & $24(33 \%)$ \\
\hline $\operatorname{Tics}(n=69)$ & $19(28 \%)$ \\
\hline Aggression/agitation $(n=71)$ & $19(27 \%)$ \\
\hline Hyperactivity & $11(15 \%)$ \\
\hline Autonomic dysfunction $(n=58)$ & $8(13 \%)$ \\
\hline Persistent focal neurologic deficits & $3(4 \%)$ \\
\hline Seizure $(n=71)$ & $1(1 \%)$ \\
\hline Transient neurologic deficits/TIA & $1(1 \%)$ \\
\hline
\end{tabular}

Legend: confidence interval (CI), Down syndrome (DS), Down syndrome regression disorder (DSRD), interquartile range (IQR) transient ischemic attack $(\mathrm{TIA}){ }^{a}=$ age at data collection, ${ }^{\mathrm{b}}=$ any non-life threatening bacterial or viral infection (e.g., upper respiratory tract infection, urinary tract infection, or pneumonia)

Table 2: Serum and Neurodiagnostic studies 


\begin{tabular}{|c|c|c|c|c|}
\hline Study & $\begin{array}{l}\text { DSRD Results Abnormal } \\
(n, \%)\end{array}$ & $\begin{array}{l}\text { DS Results } \\
\text { Abnormal } \\
(\mathrm{n} / \mathrm{N}, \%)\end{array}$ & $\mathrm{p}_{\text {Value }}$ & $95 \% \mathrm{Cl}$ \\
\hline \multicolumn{5}{|l|}{ Serum Analysis } \\
\hline ANA & $9(13 \%)$ & $21 / 422(5 \%)$ & 0.01 & $1.20-5.26$ \\
\hline Anti-DnaseB & $0(0 \%)$ & & & \\
\hline ASO $(n=58)$ & $7(12 \%)$ & & & \\
\hline B12 level & $2(3 \%)$ & & & \\
\hline Celiac panel & $5(7 \%)$ & & & \\
\hline Complete metabolic panel & $0(0 \%)$ & & & \\
\hline $\operatorname{CRP}(n=62)$ & $6(10 \%)$ & & & \\
\hline dsDNA $(n=61)$ & $0(0 \%)$ & & & \\
\hline $\operatorname{ESR}(n=66)$ & $12(18 \%)$ & & & \\
\hline Infectious screen ${ }^{a}$ & $0(0 \%)$ & & & \\
\hline Neopterin $(n=42)$ & $9(21 \%)$ & & & \\
\hline Methylmalonic acid $(n=51)$ & $1(2 \%)$ & & & \\
\hline Neurometabolic studies* $(n=32)$ & $0(0 \%)$ & & & \\
\hline Thyroid dysfunction (untreated) & $2(4 \%)$ & $160 / 842(19 \%)$ & 0.01 & $0.04-0.58$ \\
\hline TPO antibodies $(n=43)$ & $25(37 \%)$ & $110 / 478(23 \%)$ & 0.02 & $1.06-2.16$ \\
\hline Thyroglobulin antibodies $(n=42)$ & $20(30 \%)$ & $107 / 465(23 \%)$ & 0.37 & $0.80-1.82$ \\
\hline Vitamin D (median, IQR) & $26.5(15-34)$ & $39(32-47)$ & $<0.001$ & $\begin{array}{l}10.57- \\
16.9\end{array}$ \\
\hline Cytokine analysis $(n=50)$ & $20(40 \%)$ & & & \\
\hline TNF-alpha & $1(5 \%)$ & & & \\
\hline$I L-2$ & $0(0 \%)$ & & & \\
\hline slL-2 receptor & $13(62 \%)$ & & & \\
\hline IL 12 & $0(0 \%)$ & & & \\
\hline Interferon gamma & $1(5 \%)$ & & & \\
\hline IL-4 & $0(0 \%)$ & & & \\
\hline$I L-5$ & $0(0 \%)$ & & & \\
\hline IL-10 & $5(24 \%)$ & & & \\
\hline$I L-13$ & $0(0 \%)$ & & & \\
\hline$I L-17$ & $0(0 \%)$ & & & \\
\hline
\end{tabular}




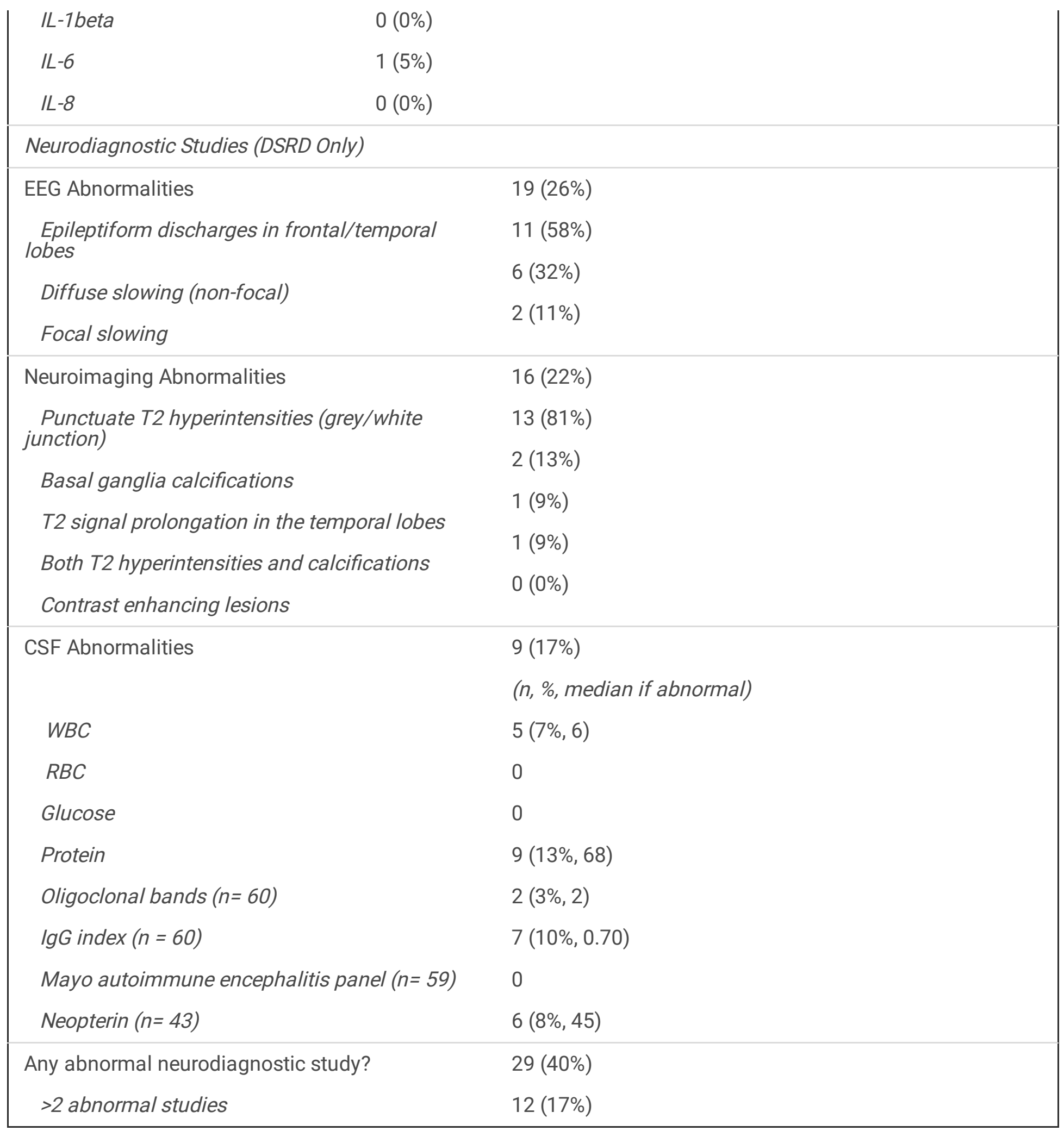

Legend: * neurometabolic studies included: lactate, pyruvate, serum amino acids, urine organic acids, free and total carnitine and acylcarnitine profiling, confidence interval $(\mathrm{Cl})$

Table 3: Therapeutic responses 


\begin{tabular}{|c|c|c|c|c|c|c|c|}
\hline \multirow[t]{2}{*}{ Therapy Type ${ }^{a}$} & \multirow[t]{2}{*}{$\begin{array}{l}\text { Utilization } \\
(\mathrm{n}, \%)\end{array}$} & \multicolumn{3}{|c|}{$\begin{array}{l}\text { Effectiveness } \\
(\mathrm{n}, \%)\end{array}$} & \multicolumn{3}{|c|}{$\begin{array}{l}\text { Any Neurodiagnostic } \\
\text { Abnormality v Normal Work } \\
\text { up }\end{array}$} \\
\hline & & $\begin{array}{l}\text { All } \\
\text { Patients } \\
(n=72)\end{array}$ & $\begin{array}{l}\text { Any } \\
\text { Neurodiagnostic } \\
\text { Abnormality } \\
(n=29)\end{array}$ & $\begin{array}{l}\text { EEG/MRI/CSF } \\
\text { Normal } \\
(n=43)\end{array}$ & $\begin{array}{l}X^{2} \\
\text { value }\end{array}$ & $\mathrm{p}_{\text {Value }}$ & $\begin{array}{l}\text { Odds } \\
\text { Ratio } \\
(95 \% \mathrm{Cl})\end{array}$ \\
\hline Antidepressant & $45(63 \%)$ & $\begin{array}{l}22 \\
(49 \%)\end{array}$ & $4 / 16(25 \%)$ & $18 / 29(62 \%)$ & 5.67 & 0.02 & $\begin{array}{l}0.20 \\
(0.05- \\
0.79)\end{array}$ \\
\hline Antipsychotic & $52(72 \%)$ & $\begin{array}{l}32 \\
(61 \%)\end{array}$ & $9 / 19(47 \%)$ & $23 / 33(70 \%)$ & 2.54 & 0.12 & $\begin{array}{l}0.39 \\
(0.12- \\
1.26)\end{array}$ \\
\hline Benzodiazepines & $63(87 \%)$ & $\begin{array}{l}49 \\
(77 \%)\end{array}$ & $18 / 24(75 \%)$ & $31 / 39(79 \%)$ & 0.17 & 0.42 & $\begin{array}{l}0.77 \\
(0.23- \\
2.59)\end{array}$ \\
\hline ECT & 49 (68\%) & $\begin{array}{l}36 \\
(74 \%)\end{array}$ & $6 / 15(40 \%)$ & $30 / 34(88 \%)$ & 12.42 & 0.01 & $\begin{array}{l}0.09 \\
(0.02- \\
0.39)\end{array}$ \\
\hline $\begin{array}{l}\text { Nutritional } \\
\text { Therapy }\end{array}$ & $29(40 \%)$ & $0(0 \%)$ & $0 / 13(0 \%)$ & $0 / 10(0 \%)$ & 0 & 1.0 & $\mathrm{n} / \mathrm{a}$ \\
\hline Immunotherapy & 43 (59\%) & $74 / 120$ & $55 / 74(74 \%)$ & 19/46 (41\%) & 10.04 & $<0.001$ & $\begin{array}{l}4.11 \\
188\end{array}$ \\
\hline Steroids & $39(54 \%)$ & & $10 / 24(42 \%)$ & $4 / 15(27 \%)$ & 0.90 & 0.34 & $9.02)$ \\
\hline$I V l g$ & 43 (59\%) & $(36 \%)$ & $24 / 26(92 \%)$ & $14 / 17(82 \%)$ & 0.05 & 0.33 & 1.96 \\
\hline Anti-CD20 & $19(26 \%)$ & $\begin{array}{l}38 / 43 \\
(88 \%)\end{array}$ & $9 / 11(81 \%)$ & 0/8 (0\%) & 9.89 & 0.01 & $7.99)$ \\
\hline$M M F / A Z$ & 19 (26\%) & $\begin{array}{l}9 / 19 \\
(47 \%)\end{array}$ & 12/13 (92\%) & 1/6 (17\%) & 12.17 & 0.01 & $\begin{array}{l}2.57 \\
(0.38- \\
17.31)\end{array}$ \\
\hline & & $\begin{array}{l}13 / 19 \\
(68 \%)\end{array}$ & & & & & $\begin{array}{l}49.5 \\
(3.84- \\
638.43)\end{array}$ \\
\hline & & & & & & & $\begin{array}{l}60.0 \\
(3.10- \\
1159.84)\end{array}$ \\
\hline
\end{tabular}

a Patients may have received multiple therapeutic interventions creating a higher " $\mathrm{n}$ " with regards to treatment interventions by class

Legend: Azathioprine (AZ), cerebrospinal fluid (CSF), electroencephalogram (EEG), electroconvulsive therapy (ECT), magnetic resonance imaging (MRI), mycophenolate mofetil (MMF).

\section{Figures}




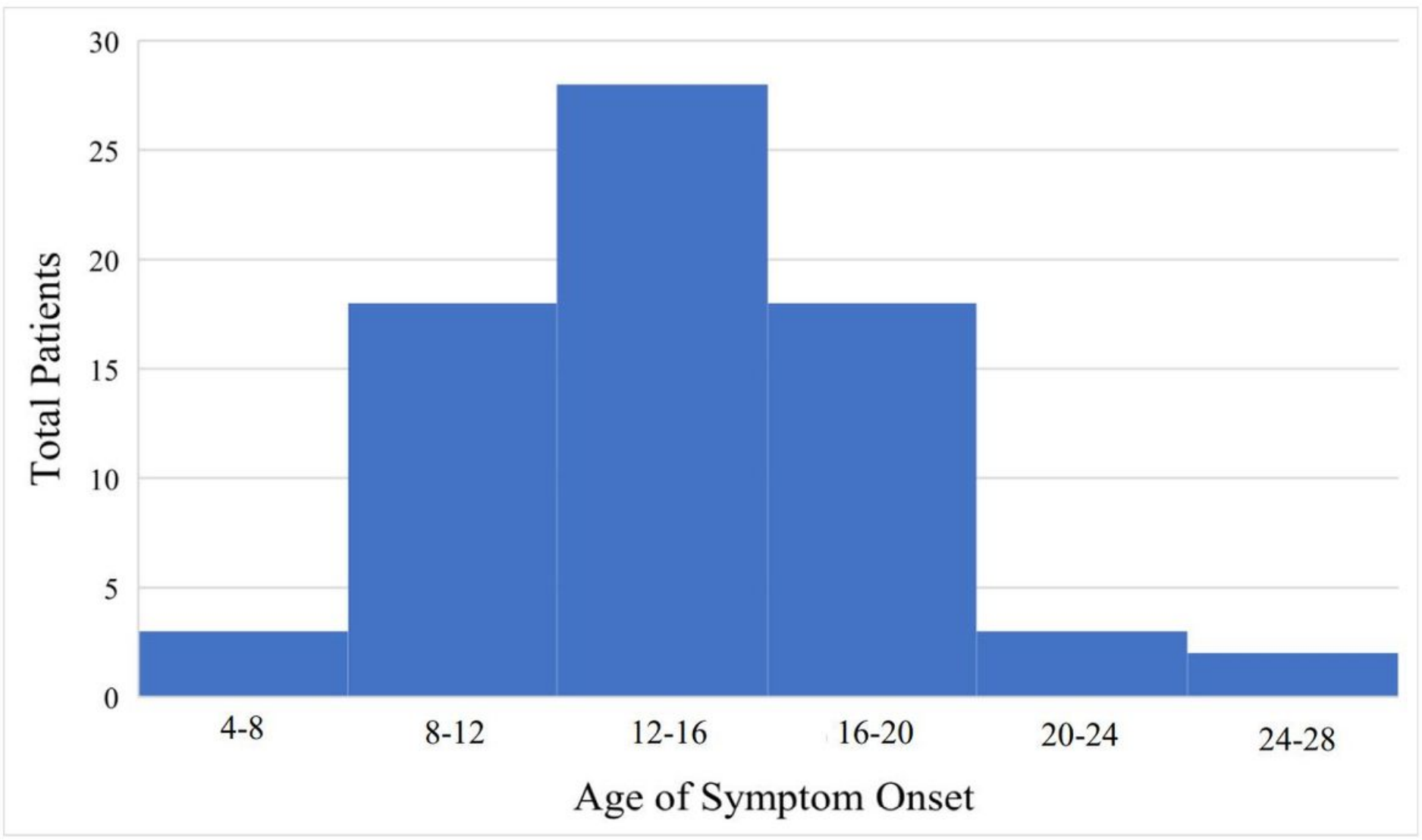

Figure 1

Histogram of symptom onset by age 


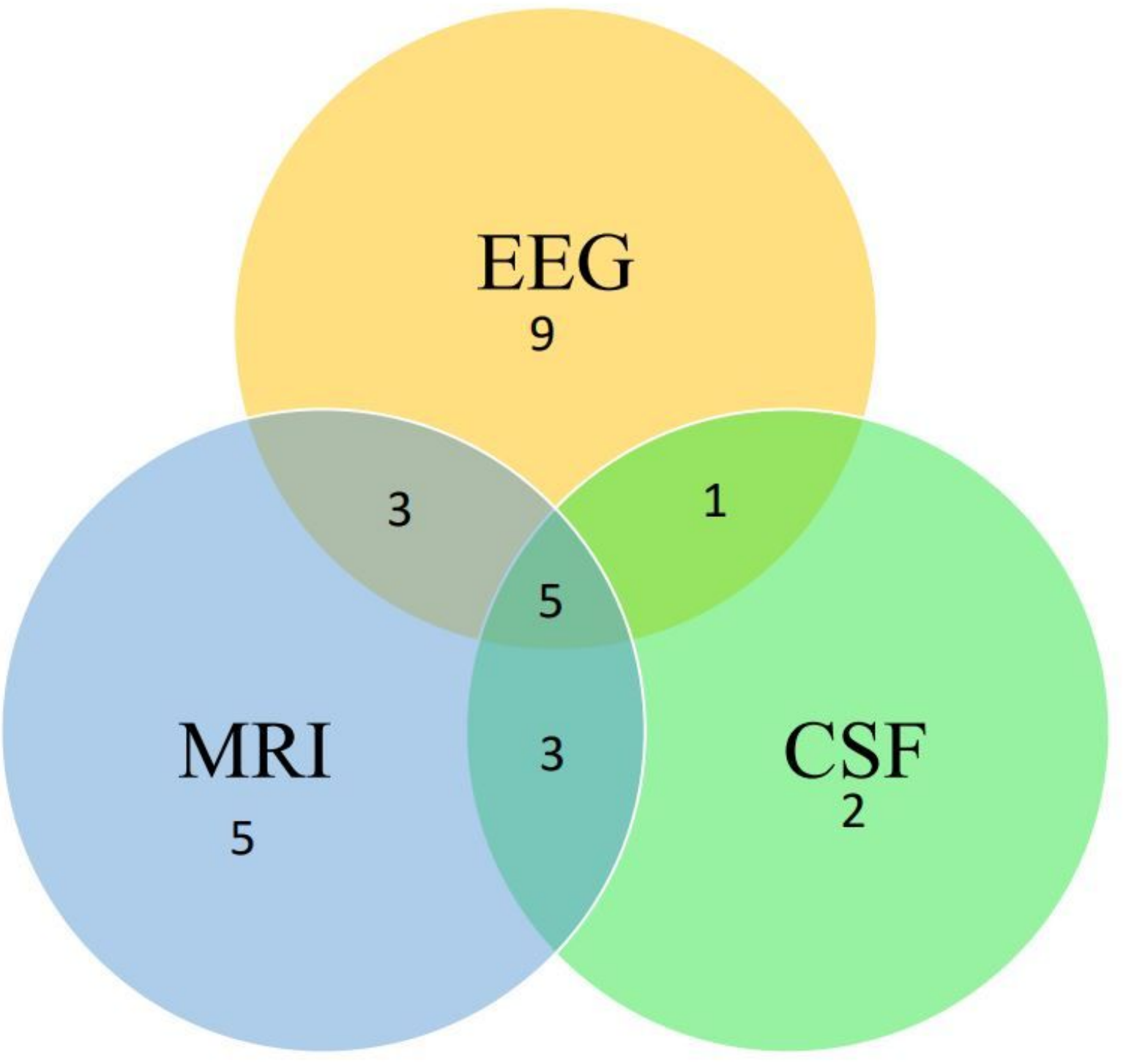

None: $n=43(60 \%)$

\section{Figure 2}

Number of patients with neurodiagnostic study abnormalities (n) 


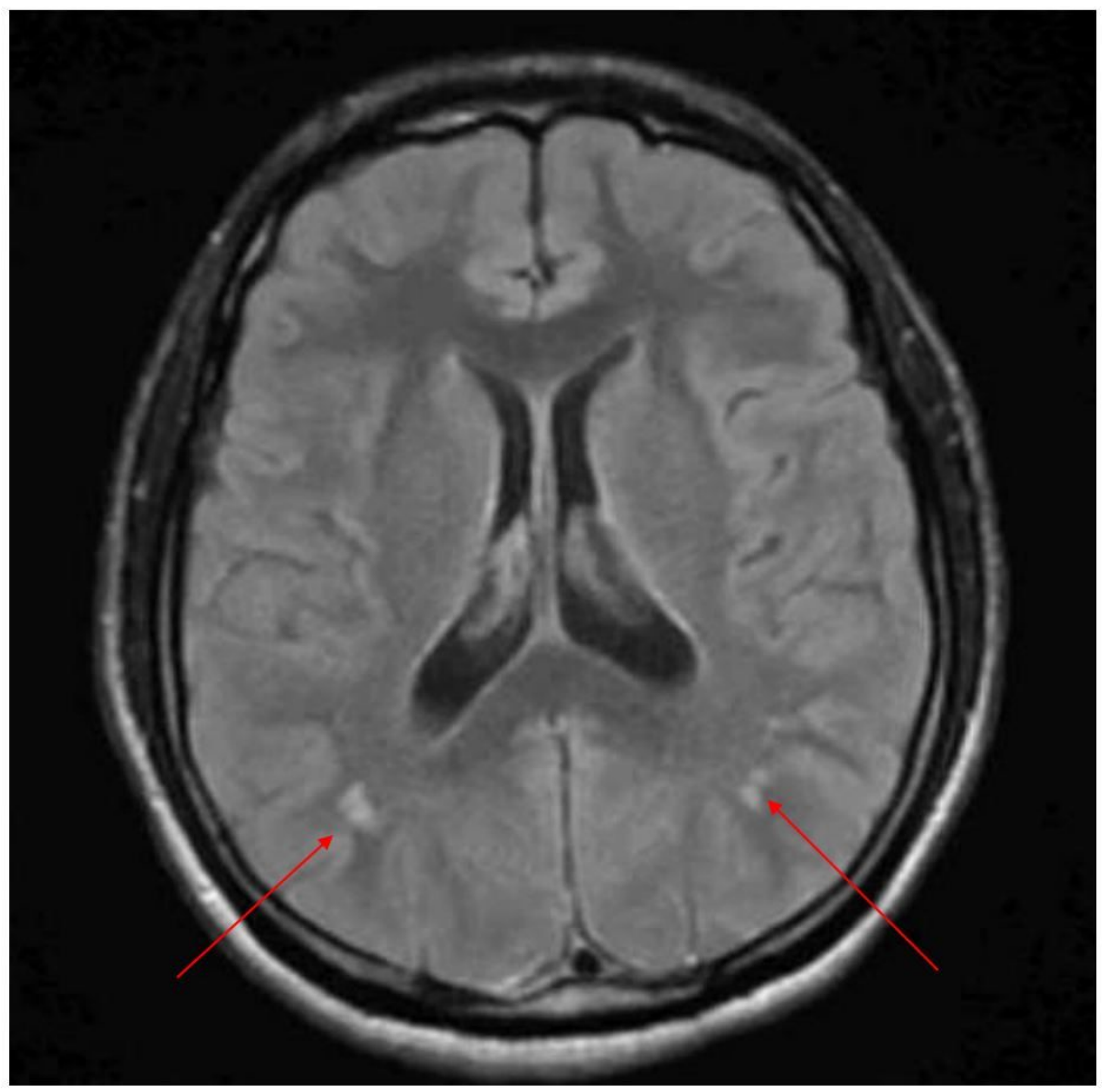

Figure 3

Axial T2 FLAIR sequence demonstrating T2 signal prolongation along the grey, white junction bilaterally. 


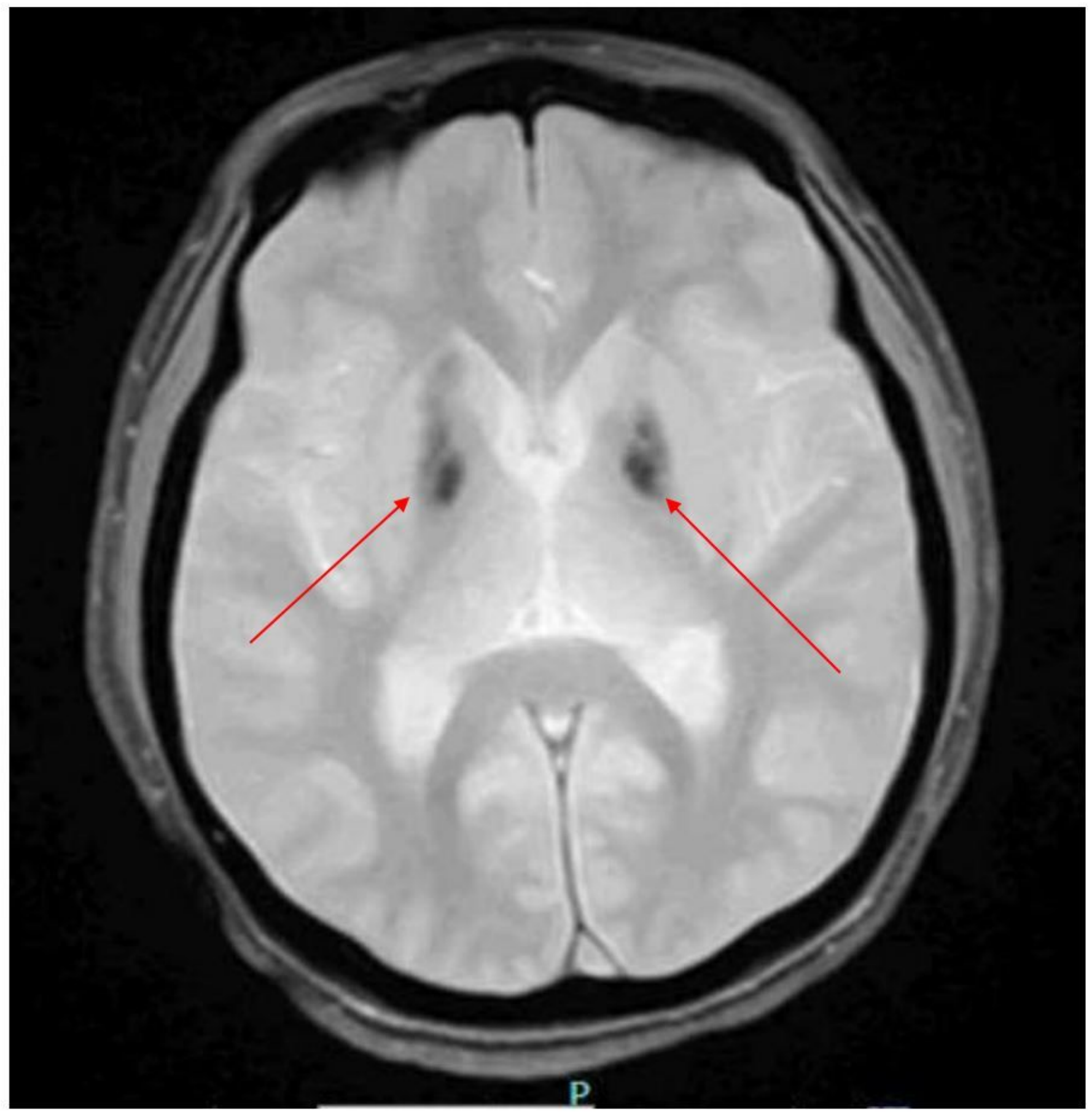

\section{Figure 4}

Axial GRE sequence demonstrating symmetric hypodensities in the bilateral deep grey nuclei consistent with calcification.

\section{Supplementary Files}

This is a list of supplementary files associated with this preprint. Click to download.

- Appendix1.docx

- Appendix2.docx 
- Appendix3.docx

- Appendix4.docx 